\title{
Percutaneous structural and valvular heart disease interventions
}

The last decade has seen an enormous leap forward in the field of percutaneous structural heart disease interventions. We have witnessed a revolutionary transformation in our ability to treat valvular and structural heart disease and are now able to offer life-saving therapies to so many patients who had few good options previously.

Severe symptomatic aortic valve stenosis (AS) in patients not deemed candidates for surgery was considered a terminal disease with an expected mortality rate worse than most cancers. The results of the pioneering PARTNER trial (1) changed the landscape in the field of the treatment of severe AS and established the technology of transcatheter aortic valve replacement (TAVR) to the field of interventional cardiology. What was once considered an impossible task of replacing a human heart valve without open heart surgery, became the standard of care for patients with severe symptomatic aortic stenosis who could not undergo surgery. What was then considered an acceptable procedure reserved only for those who could not undergo surgery, has now become the first-line standard of care treatment for the vast majority of patient with aortic stenosis needing valve replacement, regardless of surgical risk. Improvement in procedural experiences and technological advances have now made the procedure incredibly safe and effective with results far superior to the surgical outcomes that were the gold standard for decades.

Since the approval of TAVR, the field has accelerated with innovations like transcatheter mitral valve repair (TMVr), left atrial appendage occlusion, the approval of patent foramen ovale closure for cardioembolic stroke, and now transcatheter mitral valve replacement (TMVR) and percutaneous tricuspid valve repair. The successful results of the COAPT trial (2) for the first time demonstrated that reducing functional mitral regurgitation in patients with left ventricular systolic failure can improve heart failure symptoms and mortality; something surgical mitral valve repair in this cohort failed to ever demonstrate.

Despite these major advances, it is important to recognize that this seems to be just the beginning of this journey. The recently published RECOVERY trial (3) suggests that aortic valve replacement may not be just a reactionary option in the future, but can be offered even to those who have not yet developed symptoms from severe AS, as a way to help deter the downstream negative impacts of aortic stenosis. Awareness and dissemination of this success in the field is crucial for maximizing the public health impact of this great innovation.

The impact of the minimally invasive treatment options to treat structural heart disease has consolidated itself as one of the most impactful and trailblazing scientific advancements in the last 2-3 decades and has led to the emergence of a new field in cardiovascular medicine that we call structural heart disease. Through this special issue of Cardiovascular Diagnosis \& Therapy, we recognize the huge successes in this field with articles from experts on a wide variety of emerging innovations in the field of percutaneous structural heart disease treatments. We present articles on the current state of transcatheter structural interventions as well as a look into the future of some of the emerging technologies and devices of this rapidly evolving field. Lastly, we provide a perspective on training in structural heart disease from two recent graduates of structural heart disease training programs, as we must ensure adequate training within the field to ensure its continued growth and success.

\section{Acknowledgments}

None.

\section{Footnote}

Conflicts of Interest: The authors have no conflicts of interest to declare.

Ethical Statement: The authors are accountable for all aspects of the work in ensuring that questions related to the accuracy or integrity of any part of the work are appropriately investigated and resolved. 


\section{References}

1. Leon MB, Smith CR, Mack M, et al. Transcatheter aortic-valve implantation for aortic stenosis in patients who cannot undergo surgery. N Engl J Med 2010;363:1597-607.

2. Stone GW, Lindenfeld J, Abraham WT, et al. Transcatheter Mitral-Valve Repair in Patients with Heart Failure. N Engl J Med 2018;379:2307-18.

3. Kang DH, Park SJ, Lee SA, et al. Early Surgery or Conservative Care for Asymptomatic Aortic Stenosis. N Engl J Med 2020. doi: 10.1056/NEJMoa1912846.

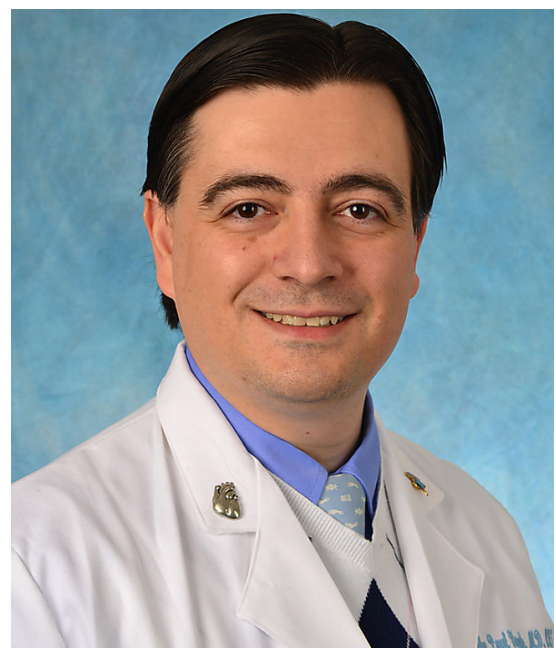

John P. Vavalle

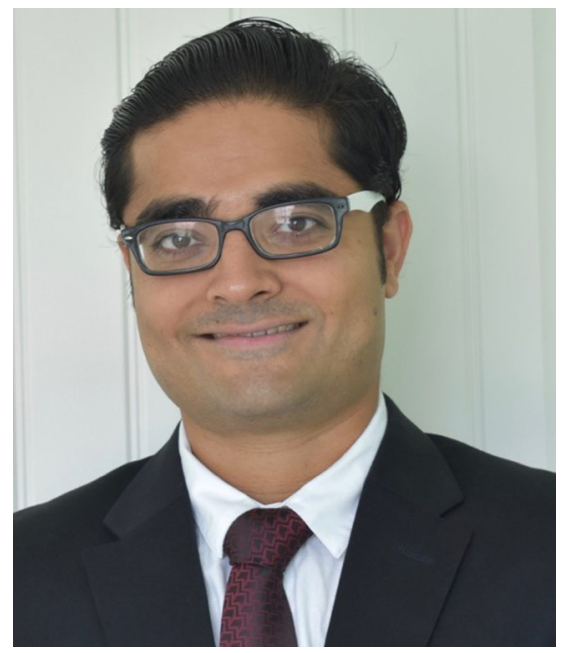

Sameer Arora

John P. Vavalle, MD, MHS, FACC Assistant Prof of Medicine, Director, Structural Heart Disease Program, University of North Carolina at Chapel Hill, Chapel Hill, NC, USA.

(Email:john_vavalle@med.unc.edu)

Sameer Arora, MD

Cardiovascular Disease Fellow, University of North Carolina at Chapel Hill, Chapel Hill, NC, USA.

(Email:saror@email.unc.edu; sameerarora.cj@gmail.com) doi: $10.21037 /$ cdt.2020.01.01

View this article at: http://dx.doi.org/10.21037/cdt.2020.01.01

Cite this article as: Vavalle JP, Arora S. Percutaneous structural and valvular heart disease interventions. Cardiovasc Diagn Ther 2020;10(1):1-2. doi: 10.21037/cdt.2020.01.01 